\title{
Characterization of magnetic colloids by means of magnetooptics
}

\author{
L. Baraban ${ }^{\mathrm{a}}$, A. Erbe, and P. Leiderer \\ Physics Department, University of Konstanz, D-78467, Konstanz, Germany
}

Received 20 February 2007

Published online: 31 May 2007 - C EDP Sciences / Società Italiana di Fisica / Springer-Verlag 2007

\begin{abstract}
A new, efficient method for the characterization of magnetic colloids based on the Faraday effect is proposed. According to the main principles of this technique, it is possible to detect the stray magnetic field of the colloidal particles induced inside the magnetooptical layer. The magnetic properties of individual particles can be determined providing measurements in a wide range of magnetic fields. The magnetization curves of capped colloids and paramagnetic colloids were measured by means of the proposed approach. The registration of the magnetooptical signals from each colloidal particle in an ensemble permits the use of this technique for testing the magnetic monodispersity of colloidal suspensions.
\end{abstract}

PACS. 82.70.Dd Colloids - 78.20.Ls Magnetooptical effects

\section{Introduction}

The interest in investigating colloidal systems has been growing during the last years because of their applications in diverse areas of research. In fundamental physics the interactions between colloids and their phase behaviour are of particular interest, since they resemble the behaviour of atoms and molecules [1]. The melting and freezing processes of "real crystals" and also atomic or molecular selfarrangement become directly visible and much more convenient to study due to the colloidal micron or sub-micron sizes [2-4]. The definition of the physical properties of the particles (magnetic moment, electric conductivity, density) during the fabrication opens possibilities for investigating a wide spectrum of interparticle interactions. Furthermore, the spherical symmetry of the particles allows their usage as an effective model to simplify the description of complicated intermolecular interactions $[5,6]$.

Nowadays, there are many experimental examples in which an ensemble of magnetic colloids reveals crystal-like behaviour in accordance with their properties and external conditions [7]. The phase behaviour of magnetically interacting colloids in an external periodic potential provided by laser tweezers has been studied in reference [8]. The system with those particles is defined by the combined effects of their magnetostatic repulsion and the trapping forces of the light potential. Classical two-stage melting of paramagnetic colloidal crystals in two dimensions is described in reference [4]. These experiments serve as a very apt demonstration of diverse applications of magnetic colloids for the understanding of phenomena which occur in real crystals. This variety of applications calls for the develop-

\footnotetext{
a e-mail: larysa.baraban@uni-konstanz.de
}

ment and improvement of methods for a comprehensive characterization of the magnetic colloidal particles.

Conventional techniques for the magnetic characterization of colloidal particles rely on ensemble properties [911]. In this work we propose a magnetooptical approach that allows for the characterization of individual particles. The magnetooptic techniques based on the Faraday effect and the magnetooptic Kerr effect usually are used for visualization of magnetic domains and measurements of the hysteresis loop [12]. Investigation of magnetic colloidal particles by the magnetooptical Kerr effect (MOKE) is presented in reference [10]. This method allows the registration of a reflected integrated signal that is the response of the ensemble of colloids on the sample. A new technique based on the X-ray magnetic circular dichroism effect for studies of magnetic structures with spatial resolution of $50 \mathrm{~nm}$ was recently proposed [13].

In this paper a modified magnetooptic technique based on the Faraday effect is described. It is a convenient method for the investigation of magnetic properties of individual colloidal particles with micrometer size and characterization of large particle arrays as well.

\section{Experimental technique}

\subsection{Fabrication and characteristics of magnetic colloids}

Two types of magnetic colloidal particles were characterized using the magnetooptic technique. The first system is given by particles coated by magnetic layers and the second one by colloidal particles consisting of magnetic bulk material. 


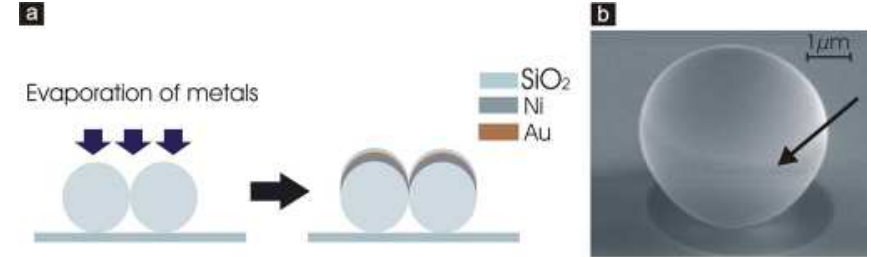

c

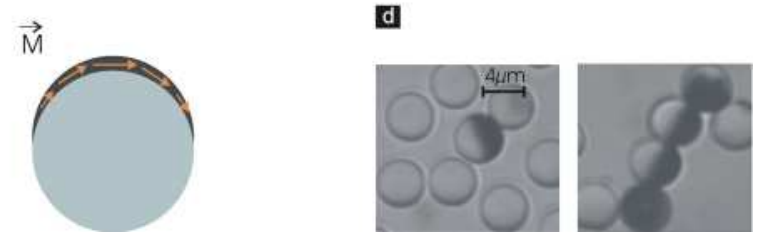

Fig. 1. (a) Fabrication procedure of capped colloids; (b) SEM image of a silica colloidal particle covered by films of $\mathrm{Ni}(50 \mathrm{~nm}), \mathrm{Au}(20 \mathrm{~nm}), \mathrm{SiO}_{2}(20 \mathrm{~nm})$. The arrow points to the boundary between metallic film and silica. The dark circle on the substrate corresponds to the area shaded by the particle during the deposition of the metal films; (c) lateral variations of magnetic properties due to the curvature of the sphere; (d) capped colloids in suspension: optical contrast between coated and uncoated surfaces of the particle.

\section{a) "Capped" colloids}

Spheres, which are covered by magnetic films, are rather interesting systems, because they enable the tailoring of the magnetic characteristics of the particles during preparation. To fabricate those colloids, an array of silica particles with diameter of $4.7 \mu \mathrm{m}$ is created on a glass substrate by slow evaporation of the solvent under ambient conditions. Self-organization leads to the formation of a closely packed hexagonal monolayer of spheres [14]. Magnetic caps were produced by the evaporation of a Ni film $(20-80 \mathrm{~nm})$ on top of the array of silica colloids at a pressure of $1 \cdot 10^{-6}$ mbar (Fig. 1(a)). In order to prevent oxidation of the Ni surface, a gold film $(20 \mathrm{~nm})$ was evaporated on top. A scanning electron microscopy (SEM) image of a capped colloid is presented in Figure 1(b).

Polycrystalline Ni provides an in-plane magnetic moment of the particles, whose value is defined by the thickness of the deposited ferromagnetic Ni film. As was shown in reference [10] the curvature of the particles can play an important role: magnetic properties of capped colloids undergo changes due to the reduction of the thickness of the magnetic material along the particles' surface (Fig. 1(c)).

The employed method of fabrication of capped particles opens possibilities for the tailoring of their properties. This can be realized by a variation of the composition of the magnetic caps during the process of deposition. The absolute value and the direction of the vector of the magnetic moment can be tuned in such a way.

The flexibility and variability of the properties of the capped colloids allow to study and to describe different kinds of interactions and arrangements in colloidal systems. The existence of a sharp optical contrast between nonmetallized and coated parts of the capped colloids (as shown in Fig. 1(d)) is of great importance. This detail can be used to visualize magnetic interactions between particles in a $2 \mathrm{D}$ colloidal crystal. Namely, the positions of the
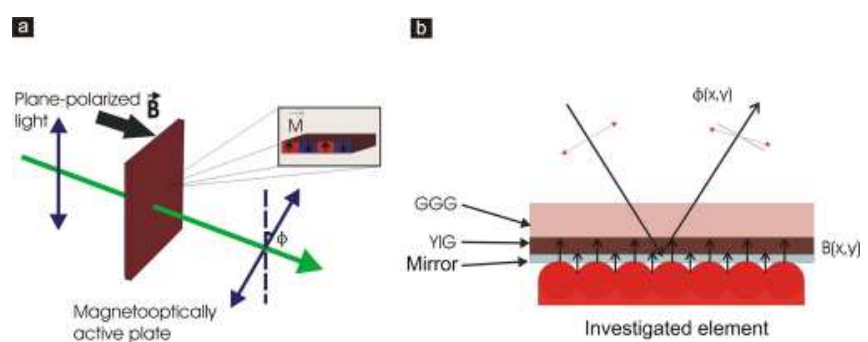

Fig. 2. (a) Sketch for the standard method of observation of the Faraday effect; (b) modified configuration of the Faraday effect for the investigation of magnetic colloids.

caps with respect to each other represent the direction of the magnetic moments on neighbouring sites of the crystal lattice. However, this application of capped colloids is possible only in the case of out-of-plane magnetic moments of the caps: the directions of magnetic moments and particles' caps coincide.

\section{b) Paramagnetic colloids}

The second type of investigated particles are polystyrene colloids doped by superparamagnetic nano-crystallites of magnetite $\left(\mathrm{Fe}_{3} \mathrm{O}_{4}\right)$ [15]. The magnetic moments of the crystallites are randomly distributed in space leading to paramagnetic behaviour of the particle. According to Langevin's theory [16], the application of an external magnetic field $B$ generates a magnetic dipole moment of the spheres. Accurate characterization of such particles is a highly relevant problem, because paramagnetic colloids have found many applications as model systems $[4,8]$.

\subsection{Measurement technique}

The polarization plane of linearly polarized light passing through a magnetized medium rotates. This is the basis of the Faraday effect, schematically shown in Figure 2(a). Traditionally this magnetooptical (m-o) effect is used for the observation of domains of thin films. Since this effect is based on the transmission of light through the media, it visualizes a magnetic structure only for films which are transparent in the visible range. Note, that only magnetic structures which reveal a component of magnetization perpendicular to the surface of the film (inset in Fig. 2(a)) can be studied. The Faraday rotation is defined as

$$
\phi(x, y)= \pm V(\lambda) \times b(x, y) \times l,
$$

where $\phi(x, y)$ is the distribution of the angle of rotation, $V(\lambda)$ is the Verdet constant of the material, $b(x, y)$ is the distribution of the local magnetic field inside the m-o active layer, and $l$ is the length of light propagation in the $\mathrm{m}-\mathrm{o}$ active layer. The sign of the angle of rotation depends on the direction of the local magnetization in the film with respect to the direction of light propagation.

Investigations of magnetic properties of colloidal particles require some changes in the conventional method, which usually is applied for studying thin film magnetism, 


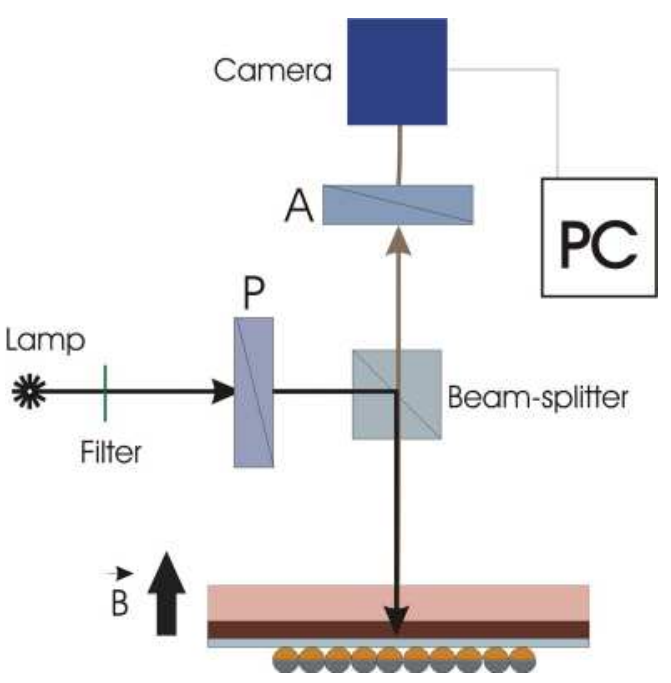

Fig. 3. Schematic diagram of the setup. A CCD-camera registers the transmitted intensity in the system polarizer-sampleanalyzer.

due to the following reasons: a) the curvature of the particles causes undesirable scattering of light, which leads to a decrease of the signal level; b) paramagnetic colloids and metallic caps are not transparent in the visible range.

Therefore, an experimental setup based on the reflection of light by a mirror layer is used. Such a configuration of the experimental setup allows to avoid the foregoing problems for the observation of the magnetic signal coming from the colloids. The investigated element (magnetic colloids) is in close contact with the rather thin $(20 \mathrm{~nm})$ reflecting layer of gold as is shown in Figure 2(b). The thickness of the mirror layer can be varied to improve the m-o contrast. It should be noted that in the case of capped colloids the contact of magnetic caps with the mirror is necessary. The m-o active thin film $(2 \mu \mathrm{m})$ is yttrium iron garnet (YIG) grown on a gallium gadolinium garnet (GGG) with the magnetic sensitivity of $10 \mu \mathrm{T}$. The investigated element creates a spatial map of the local magnetic field $b(x, y)$ inside the magnetooptical medium. A polarized beam propagates through the YIG film. Reflected by the mirror, light carries the spatial distribution of Faraday rotation $\phi(x, y)$ to the analyzer. The transmitted intensity in the system polarizer-sample-analyzer is described by the Mallus law: $I(x, y)=I_{0} \sin ^{2}(\alpha+\phi(x, y))$, where $I_{0}$ is the intensity of the incident light and $\alpha$ is the angle between the analyzer and the polarizer. The lateral resolution of the technique is approximately $2 \mu \mathrm{m}$ and it is limited by the thickness of the garnet film. Thus, the thickness of the m-o layer limits also the minimal size of particles which can be studied $(1-2 \mu \mathrm{m})$. A similar construction was used in experiments on superconductor films, which are described in [17]. In the case $\alpha=0$ the method has an obvious disadvantage, because of the quadratic dependence of the intensity on the angle at small values of $\phi$. Therefore, it is impossible to get information about the sign of the Faraday rotation. This problem, however, can be avoided by setting a small mismatch between polarizer and analyzer. In our experiments small $\left(4-5^{\circ}\right)$ values of $\alpha$
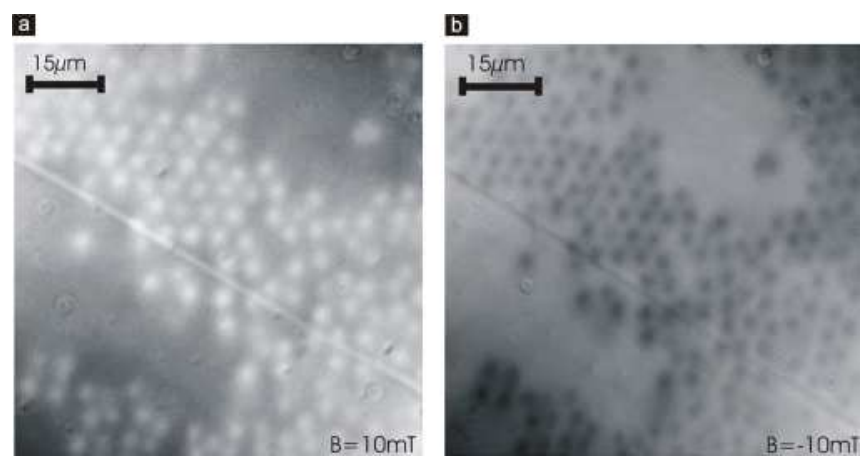

Fig. 4. Magnetooptical images of Ni-capped colloids with diameter $4.7 \mu \mathrm{m}$; (a) $B=10 \mathrm{mT}$; (b) $B=-10 \mathrm{mT}$.

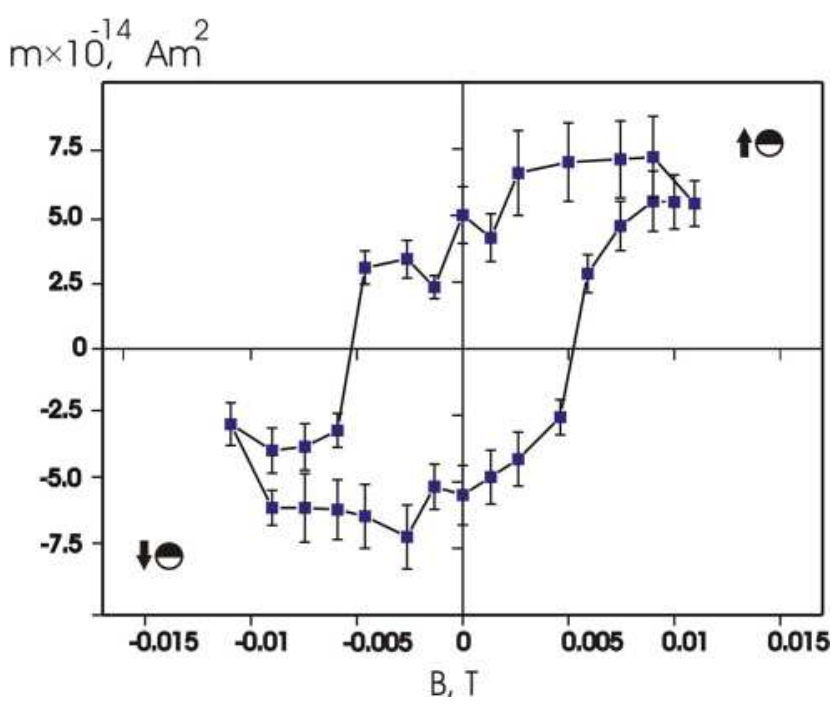

Fig. 5. Hysteresis loop obtained for Ni-capped colloids with diameter $4.7 \mu \mathrm{m}$ in a vertically applied magnetic field.

were used. A m-o image is obtained as a result of three measurements at different angles $\left(\alpha= \pm \alpha^{\prime}, \alpha=0\right)$. Such an approach allows the determination of $\phi$ in each pixel of the image, taking into account the sign of the Faraday rotation. It should be noted that the contrast of the observed magnetic structure depends essentially on $\alpha$, and that the sensitivity of the method at small applied magnetic fields is raised by these differential measurements.

\subsection{Experimental setup}

A sketch of the experimental setup is shown in Figure 3. Illumination from the white-light source passes through the filter (which transmits light at wavelength $\lambda=532 \mathrm{~nm}$ ) and polarizer $(\mathrm{P})$. A Glan-Thompson polarizer (extinction ratio of $10^{-6}$ ) is used in our experimental setup in order to improve the contrast of the visualized structure [18]. The polarized light is partly reflected by a Smith beam splitter $[18,19]$ to the sample, where it propagates through the active layer (YIG) and is reflected by the mirror. The magnetic field is applied perpendicularly to the plane of the sample. Light with spatially changed polarization passes 

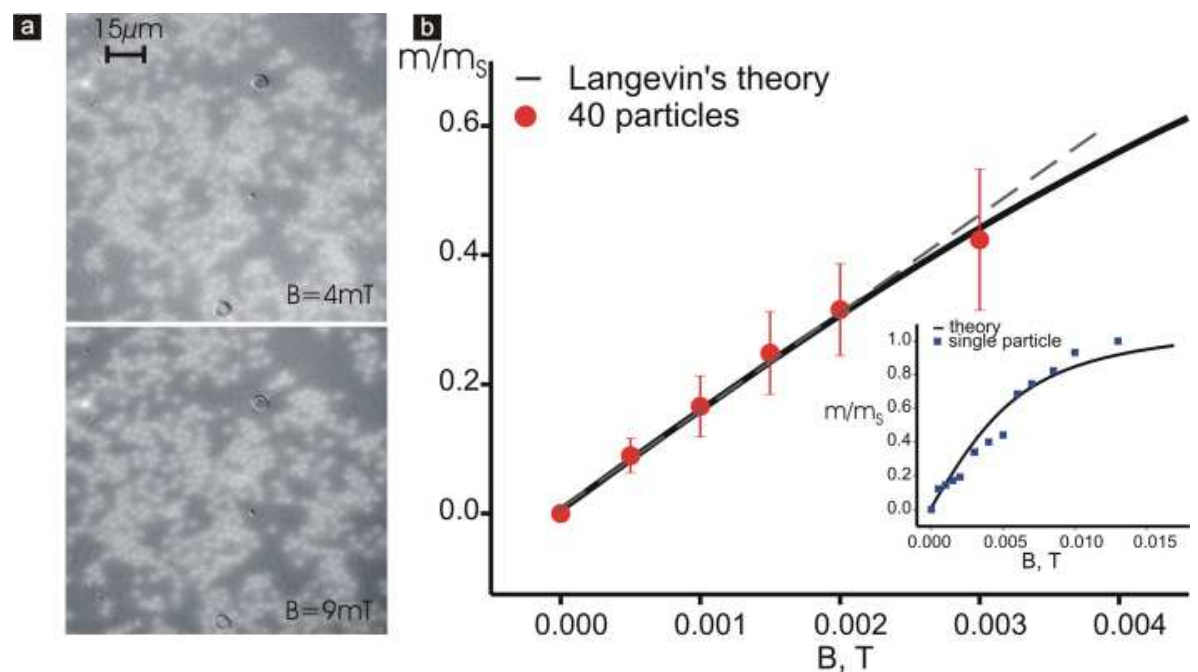

Fig. 6. Magnetooptic measurements with paramagnetic particles. (a) Images of colloids with diameter $4.5 \mu \mathrm{m}$ taken at $4 \mathrm{mT}$ and $9 \mathrm{mT}$; (b) linear range of the magnetization curve: the points are obtained from the m-o experiment for an ensemble of 40 particles (circles); the black solid line is a theoretical result in accordance with Langevin's theory $\left(M=M_{0}\left(\operatorname{coth}\left(\alpha B-\frac{1}{\alpha B}\right)\right)\right.$, $M_{0}=1, \alpha=378$ ); the inset in panel (b) displays the magnetization curve measured for a single particle (squares).

through the analyzer (A) and a 16-bit CCD-camera registers the spatial distribution of the transmitted intensity. Therefore, the "spatial map" of the angle of rotation can be defined as

$$
\phi(x, y)=\frac{\left(I_{+}-I_{-}\right)}{4 \alpha I_{0}},
$$

where $I_{+}, I_{-}, I_{0}$ are the intensities of light transmitted by the analyzer at different angles between polarizer and analyzer. In accordance with equation (1) the spatial map of $\phi(x, y)$ gives a distribution of the local magnetic field $b(x, y)$ induced by the stray field from the magnetic colloids in the m-o layer, from which the magnetization and magnetic moment of the particles can be calculated.

\section{Results}

The magnetic moments of colloids and the magnetization curve were measured by means of the described technique.

\section{a) "Capped" colloids}

In Figure 4 m-o images with Ni-capped colloidal particles with $4.7 \mu \mathrm{m}$ diameter are presented. The distribution of the intensity in the picture displays a spatial distribution of the signal, transmitted by the analyzer. These images provide information about the transformation of the polarization of light inside the active layer (YIG). The magnetic moments of the magnetized colloids change the local magnetic moment in the m-o layer at the positions of the colloids. The bright and dark spots in the pictures correspond to the different signs of the Faraday rotation due to the direction of the application of the external magnetic field.

The measurements allow to estimate magnetic properties of capped colloids, and to compare them with characteristics of bulk Ni. Figure 5 displays the transmitted signal, which was taken at different magnetic fields from colloids coated with polycrystalline Ni films with thickness $80 \mathrm{~nm}$. The curve is an averaged result for an ensemble of 160 particles. These data allow us to evaluate the magnetization and magnetic moments of the colloids in the saturation regime and to define the coercivity field as well. We observed a value of magnetisation in saturation of $M_{s}=4.5 \cdot 10^{4} \mathrm{~A} / \mathrm{m}$, which is comparable to the magnetisation of $\mathrm{Ni}$ at room temperature [20], but could be slightly underestimated due to heating influence of the setup. An estimated value of the magnetic moment of the colloidal particles is equal to $m_{s}=8 \cdot 10^{-14} \mathrm{Am}^{2}$. In addition, the hysteresis loop can characterize the stability of the magnetic moments of the particles, in accordance with the values of remanent magnetization and coercivity field. The magnetic softness of the caps is caused by the polycrystalline behaviour of the evaporated Ni films.

\section{b) Paramagnetic colloids}

In Figure 6 results of the m-o measurements for polystyrene colloids with diameter $4.5 \mu \mathrm{m}$ doped by magnetite are shown. The m-o images (Fig. 6(a)) were taken at an applied magnetic field of 4 and $9 \mathrm{mT}$. Since the colloids under investigations are paramagnetic, increasing of the magnetic field increases the magnetic moments of the particles. The growth of magnetic moments of the colloids leads to a rise in the contrast between the signals from background and particles. The averaged result which characterizes an ensemble of 40 particles is presented in Figure 6(b). The agreement between the measured data and Langevin's theory is excellent in the linear part of the magnetization curve. Most applications and experiments are based on the particles' properties in this regime. We can, however, characterize the particles at higher magnetic fields as well, as is shown in the inset of Figure 6(b) for a single particle. The measured data agree with the theoretical model within the accuracy of the experiment. 
The value of the magnetic moment of the single paramagnetic particle in saturation is determined from the measured magnetization curve $\left(m_{s}=3 \cdot 10^{-13} \mathrm{Am}^{2}\right)$, which is comparable with the value measured earlier [11,21]. A linear fit in Figure 6(b) was used to estimate the effective susceptibility of a colloidal particle $\chi=3.4 \mathrm{Am}^{2} / \mathrm{T}$, which is also in agreement with the results from experiments described in [11].

It is important to note that most of the techniques proposed earlier for the characterization of paramagnetic colloids did not give results for individual particles, while the modified m-o technique allows to determine magnetic properties of single particles and to carry out a comparative analysis of magnetooptical signals from the whole ensemble of colloids. It should be mentioned that m-o signals from individual particles and from single colloids, which are chosen from an ensemble, will be different (10-15\%). The measured intensity of particles in an array will be therefore slightly overestimated, because of the influence of the magnetic field of the neighbours.

\section{Conclusions and outlook}

In this work we have investigated the possibility to study the magnetic properties of paramagnetic colloids and colloids with half-spherical magnetic caps by means of a magnetooptic technique. It has been shown that the described approach allows the determination of magnetic moments and measurements of magnetization curves of individual particles, providing characterization of colloids in a wide range of the magnetic field. The resolution provided by the proposed technique allows to register the deviations of m-o signals from particle to particle. This important detail distinguishes the m-o method of characterization of magnetic colloids from other approaches, because it opens possibilities in the characterization of large particle arrays and in the analysis of the magnetic monodispersity of the suspensions. Those studies require a strict division between signals from the investigated elements (colloids) and from background (YIG, illumination). Indeed, the analysis of the homogeneity of the magnetooptical signals from particles is a subject of future researches. Such a comprehensive characterization of magnetic colloids enables to visualize and to describe different kinds of interactions and arrangements in colloidal systems.
We gratefully acknowledge the support of the IRTG Soft Condensed Matter Physics of Model Systems. We would like to thank B.-U. Runge and B. Biehler for useful discussions.

\section{References}

1. P.M. Chaikin, T.C. Lubensky, Principles of Condensed Matter Physics (Cambridge University Press, 1995).

2. A.B. Schofield, P.N. Pusey, P. Radcliffe, Phys. Rev. B 72, 031407 (2005).

3. E.V. Shevchenko, D.V. Talapin, N.A. Kotov, S. O'Brien, C.B. Murray, Nature 439, 55 (2006).

4. K. Zahn, R. Lenke, G. Maret, Phys. Rev. Lett. 82, 2721 (1999).

5. M.E. Leunissen, C.G. Christova, A.-P. Hynninen, C.P. Royall, A.I. Campbell, A. Imhof, M. Dijkstra, R. van Roij, A. van Blaaderen, Nature 437, 235 (2005).

6. P.J. Lu, J.C. Conrad, H.M. Wyss, A.B. Schofield, D.A. Weitz, Phys. Rev. Lett. 96, 028306 (2006).

7. W. Wen, L. Zhang, P. Sheng, Phys. Rev. Lett. 85, 5464 (2000).

8. K. Mangold, P. Leiderer, C. Bechinger, Phys. Rev. Lett. 90, $158302(2003)$.

9. V. Maceira, M. Spasova, M. Farle, Adv. Funct. Mater. 15, 1036 (2005).

10. M. Albrecht, G. Hu, I.L. Guhr, T.C. Ulbrich, J. Boneberg, P. Leiderer, G. Schatz, Nat. Mater. 4, 195 (2005).

11. K. Zahn, J.M. Mendez-Alcaraz, G. Maret, Phys. Rev. Lett. 79, 175 (1997).

12. R.P. Cowburn, D.K. Koltsov, A.O. Adeyeye, M.E. Welland, Phys. Rev. Lett. 83, 1042 (1999).

13. S. Eisebitt, J. Lünning, W.F. Schlotter, M. Lörgen, O. Hellwig, W. Eberhardt, J. Stöhr, Nature 432, 885 (2004).

14. F. Burmeister, C. Schäfle, B. Keilhofer, C. Bechinger, J. Boneberg, P. Leiderer, Adv. Mater. 10, 495 (1998).

15. Dynalbeads (http://www.dynalbiotech.com).

16. Neil W. Ashcroft, N. David Mermin, Solid State Physics (Brooks Cole, 1976).

17. B. Biehler, B.-U. Runge, P. Leiderer, R.G. Mints, Phys. Rev. B 72, 2 (2005).

18. B. Biehler, PhD Thesis, Konstanz University, Germany (2005).

19. P. Goa, H. Hauglin, M. Baziljevich, E. Ill'yashenko, P. Gammel, T. Johansen, Superconduct. Sci. Technol. 14, 729 (2001).

20. R.C. O'Handley, Modern Magnetic Materials (Wiley Interscience, New York, 2000).

21. V. Blickle, D. Babic, C. Bechinger, Appl. Phys. Lett. 87, 101102 (2005). 